\title{
Time causes forgetting from working memory
}

\author{
Pierre Barrouillet • Annick De Paepe • Naomi Langerock
}

Published online: 20 December 2011

(C) Psychonomic Society, Inc. 2011

\begin{abstract}
Although forgetting in the short term is a ubiquitous phenomenon, its exact causes remain undecided. The aim of the present study was to test the temporal decay hypothesis according to which memory traces fade away with time when attention is diverted by concurrent activities. In two experiments involving complex span tasks, adults were asked to remember series of items (either letters or spatial locations) while verifying multiplications. The duration of processing was manipulated by presenting multiplications either in word (three $\times$ four $=$ twelve) or digit $(3 \times 4=12)$ format, the former taking longer to solve, while the time available to restore memory traces after each operation was kept constant across conditions. In line with the temporal decay hypothesis, the longer solution times elicited by solving word multiplications resulted in poorer recall performance. The fact that longer processing times had a comparable effect on both verbal and visuospatial memory and that the difference between conditions remained stable from the first to the last trials makes it difficult to account for these findings by assuming that forgetting is exclusively due to representationbased interference or buildup of proactive interference.
\end{abstract}

Keywords Working memory Dual-task performance $\cdot$ Short term memory $\cdot$ Memory

\section{P. Barrouillet $(\triangle) \cdot N$. Langerock}

Faculté de Psychologie et de Sciences de l'Education,

Université de Genève,

40, bd du pont d'Arve,

1211 Genève 4 , Switzerland

e-mail: pierre.barrouillet@unige.ch

\section{A. De Paepe}

Ghent University,

Sint-Pietersnieuwstraat 25,

9000 Gent, Belgium
The fact that information temporarily maintained for immediate use or recall rapidly vanishes from memory is a ubiquitous phenomenon. Surprisingly, the exact causes of this forgetting remain undecided. A venerable tradition attributed forgetting to the sheer passage of time, assuming that memory traces suffer from a temporal decay (J. Brown, 1958; Conrad, 1967; Peterson \& Peterson, 1959). However, further appraisals of the findings on which this hypothesis was based led to the conclusion that, currently, there is no evidence for temporal decay in working memory (Lewandowsky \& Oberauer, 2009; Lewandowsky, Oberauer, \& Brown, 2009). Thus, modern psychology has rejected this hypothesis and commonly assumes that forgetting is due not to decay (Brown \& Lewandowsky, 2010), but to representation-based interference created by the intervening events occurring between encoding and retrieval (Nairne, 1990; Oberauer \& Kliegl, 2006; Oberauer \& Lewandowsky, 2008).

However, some recent studies have cast doubt on the idea that there is no temporal decay in the short term by reporting facts that are difficult to explain without any effect of time per se (Cowan \& AuBuchon, 2008; Ricker \& Cowan, 2010). Moreover, within the time-based resource-sharing (TBRS) model framework, we have gathered a large body of evidence demonstrating that, in complex span tasks, recall performance is a function of the time during which intervening activities occupy attention, suggesting that memory traces suffer from a temporal decay while attention is diverted away (Barrouillet, Bernardin, \& Camos, 2004; Barrouillet, Bernardin, Portrat, Vergauwe, \& Camos, 2007; Barrouillet, Portrat, \& Camos, 2011; Vergauwe, Barrouillet, \& Camos, 2009, 2010).

The aim of the present study was to provide direct evidence that memory traces decay with time while attention is diverted by intervening activities. For this purpose, we used a complex span task paradigm in which participants are 
presented with memory items for further recall, each item being followed by a distractive task. Previous studies have shown that recall performance in this kind of span task depends on the ratio between the time taken to perform the distractive task (i.e., the processing time) and the free time available to reactivate memory traces (i.e., the refreshing time): The higher this ratio, the poorer the recall performance (Barrouillet et al., 2004, 2007; Barrouillet, Portrat, $\&$ Camos, 2011). One possible account for this relation is to assume that memory traces decay with time. Processing will prevent refreshing processes from counteracting this decay, with longer processing resulting in more forgetting. However, the fact that memory performance depends on a time ratio does not constitute definite evidence for a temporal decay of memory traces. It could be imagined that the memoranda suffer not from decay, but from representationbased interference created by encoding the distractor items and that some time-dependent refreshing processes could repair the damages created by this interference. In this case, memory performance would not depend on processing time but should increase with refreshing time (Lewandowsky \& Oberauer, 2009; Lewandowsky et al., 2009; Oberauer \& Kliegl, 2006). Thus, demonstrating that memory traces decay with time requires showing that, while refreshing time is kept constant, increasing processing time results in poorer recall performance. For this purpose, we took advantage of the fact that it takes longer to solve multiplications presented in a word (three $\times$ four) than a digit $(3 \times 4)$ format. Provided that the operands are small (here, we used operands up to a maximum of 5), there is a large consensus in the literature that it should be assumed that both types of operations are solved through the same process of retrieval from long-term memory of arithmetic facts that are stored in a verbal format (Dehaene, 1992). The longer solving times for word multiplications have been attributed to encoding differences (Noel, Fias, \& Brysbaert, 1997).

In a first experiment, participants were presented with a complex span task in which they had to remember series of consonants. After presentation of each letter, they were asked to verify three successive multiplications presented either in digits (short processing time) or in words (long processing time) and to give their response (either "correct" or "false") by pressing keys on a keyboard (Fig. 1). In order to keep refreshing times equal in both conditions, each response in the processing task was followed by a constant delay of $800 \mathrm{~ms}$ before the appearance of the next multiplication or letter. When refreshing times are kept constant, the temporal decay hypothesis predicts more forgetting and poorer recall with a longer processing time. Thus, we predicted poorer recall in the word multiplication condition.

\section{Experiment 1}

\section{Participants}

Thirty-three undergraduate students ( 27 females; mean age $=$ 20.1 years, $S D=2.1$ ) at the University of Geneva participated for partial course credit.

\section{Materials and procedure}

Participants were presented with 16 series of five consonants to be remembered that were randomly assigned to the two conditions determined by the nature of the multiplications to be verified (either in digits or in words), with 8 series per condition. Series were constructed in such a way that
Fig. 1 Trial design for the two experiments. Each memory item (letters for Experiment 1 and spatial displays for Experiment 2) was presented for $1 \mathrm{~s}$ and was followed, after a delay of $100 \mathrm{~ms}$, by three multiplications for verification. Participants gave a manual response (true or false) by pressing appropriate keys on a keyboard. The following multiplication or memory item was displayed $800 \mathrm{~ms}$ after this keypress. The figure illustrates the fact that processing times (gray lines) are longer for multiplications in words (right panel) than for those in digits (left panel)

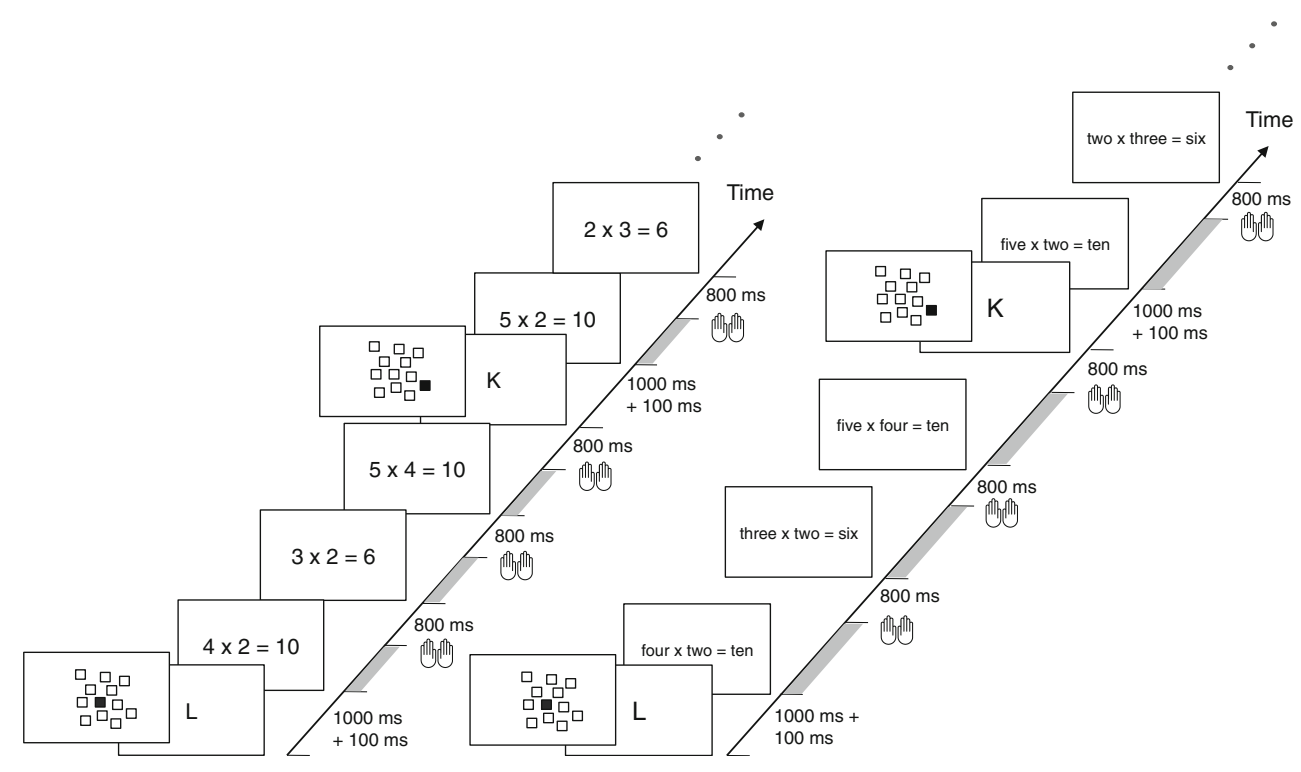


acronyms and alphabetically ordered strings were avoided. Each consonant was followed by three multiplication problems and their solution (e.g., $3 \times 5=15$ ). Both operands of the multiplication problems were smaller than or equal to 5 . Problems involving 0 or 1 and tie problems (e.g., $3 \times 3$ ) were excluded. Commuted pairs (e.g., $3 \times 5$ and $5 \times 3$ ) were considered as two different problems. Half of the problems were presented with a wrong answer selected from the correct multiplication table (e.g., $3 \times 5=10$ ). Problems containing the same components could not be displayed within the same burst of three multiplications that followed each letter. During the experimental phase, the 16 series of letters were randomly assigned to the 16 trials $(8$ per condition) that were presented in a random order.

Before each trial, participants were informed about the format of the forthcoming multiplications (words or digits). A ready signal (an asterisk) was centered on-screen for $750 \mathrm{~ms}$, followed by a white screen for $100 \mathrm{~ms}$. Then the first letter was displayed for $1,000 \mathrm{~ms}$, followed after a delay of $100 \mathrm{~ms}$ by three successive multiplications, by another letter for $1,000 \mathrm{~ms}$ and, after a delay of $100 \mathrm{~ms}$, by three other multiplications, and so on until the end of the series. Participants were asked to read aloud each letter when it appeared and to verify the answers of the multiplications as quickly as possible, without sacrificing accuracy, by pressing either the A or the L key for "correct" and "false," respectively. These keypresses removed the problem from the screen, which remained empty for $800 \mathrm{~ms}$, creating a constant delay before the presentation of the next multiplication or letter. However, if participants had not responded within a time limit of $1,000 \mathrm{~ms}$ for the digit multiplications and $1,500 \mathrm{~ms}$ for the word multiplications (these values were fixed from a pretest), the problem disappeared from screen, but participants were informed at the beginning of the experiment that their response was still recorded for a limited amount of time. In reality, the experiment did not proceed until the participant had given his or her response, which was followed by the 800 -ms delay. This was done to create the illusion that the task was totally computer paced, thus avoiding the possibility that participants would postpone the arithmetic task to reactivate memory traces. At the end of the series, the word rappel (recall) was displayed on-screen, and participants had to type the letters in correct order by filling an empty five-slot frame. The score was the number of series of letters recalled in correct order. Besides the letters recalled, reaction times and accuracy during the multiplication task were recorded.

\section{Results and discussion}

Six participants who had fewer than $80 \%$ correct responses in the multiplication task were discarded from the analyses. As we expected, the remaining 27 participants took longer to solve multiplications in word than in digits $(1,448 \mathrm{~ms}, S D=$
$219 \mathrm{~ms}$, and $944 \mathrm{~ms}, S D=124 \mathrm{~ms}$, respectively), $F(1,26)=$ $287.40, p<.001$. In line with the temporal decay hypothesis, these longer processing times resulted in poorer recall performance, with a rate of series correctly recalled of $.45(S D=.28)$ and $.63(S D=.30)$ for the word and digit conditions, respectively, $F(1,26)=35.84, p<.001$. However, verifying word multiplications involved a higher rate of errors $(10 \%, S D=$ $5 \%$, and $6 \%, S D=4 \%$, for words and digits, respectively), $F(1,26)=27.18, p<.001$, something that could have an impact on recall performance. Indeed, it has been shown that errors are usually followed by posterror processes that occupy the attentional bottleneck and delay the processing of the next stimulus (Jentzsch \& Dudschig, 2008; Laming, 1979). Because this posterror processing would take place during the refreshing periods following each multiplication, the higher number of errors committed with words could have shortened the periods of refreshing in this condition, resulting in poorer recall performance (Lewandowsky \& Oberauer, 2009). Thus, in order to equalize the rate of errors between conditions, we excluded all the trials on which a given participant committed more than one error out of the 15 multiplications, as well as those participants who had fewer than 3 remaining trials in one or both conditions after this trimming procedure had been applied. There were 19 remaining participants with a mean of 5.5 trials in the word condition and 6.7 trials in the digit condition. The mean processing times for these remaining trials were $1,448 \mathrm{~ms}(S D=252 \mathrm{~ms})$ and $918 \mathrm{~ms}(S D=115 \mathrm{~ms})$ for the word and digit conditions, respectively, $F(1,18)=$ $169.09, p<.001$. Although the rate of error no longer differed between conditions ( $3 \%$ in both condition), $F<1$, the effect on recall performance remained. The rate of series correctly recalled was still lower in the word than in the digit condition $(.54, S D=.29$, and $.74, S D=.24$, respectively), $F(1,18)=$ $25.12, p<.001$. It is worth noting that this effect was still significant when the order of recall was not taken into account $(.59, S D=.31$, and $.82, S D=.22$, for the word and digit conditions, respectively), $F(1,18)=18.73, p<.001$, indicating that the observed effect was due not to order errors, but to recall failures, as a temporal decay hypothesis would predict.

Although these results support the temporal decay hypothesis, it could be argued that they result from representationbased interference. Such an account would assume that the representations resulting from reading word numbers interfere more with the memory traces of letters than do the representations resulting from processing numbers in their Arabic format. Two reasons could be put forward. First, number words are themselves made of letters and could have created more interference than did digits. Second, letters are most probably maintained in their phonological code. Even though prominent theories assume that both digit and word formats activate the same verbal code storing arithmetic facts (Dehaene, 1992), other theories have argued that phonological representations of numbers are automatically activated from 
the graphemic representation of words, but to a lesser extent from the ideographic representation of digits (Fias, Brysbaert, Geypens, \& d'Ydewalle, 1996). Following the latter hypothesis, the phonological codes activated by number words would blur the memory traces of letters to a greater extent than would digits, thus leading to more forgetting.

To discard this alternative explanation, we replicated our findings with a memory material that does not involve phonological coding. We designed a second experiment in which the memoranda consisted not of letters, but of spatial locations defined by 12 squares. These squares were presented on-screen at random locations to avoid any possibility of verbal coding by localizing a given square by its coordinates in the matrix usually used in spatial memory tasks (Darling, Della Sala, \& Logie, 2007; see Fig. 1). Each spatial location was presented for $1 \mathrm{~s}$ by turning one of these squares blue, followed by three multiplications presented in either word or digit format. Because working memory spans are lower for spatial than for verbal information (Logie, 1995; Vergauwe et al., 2010), participants were presented with a series of only three spatial locations (and thus, nine multiplications). Because the temporal decay hypothesis assumes that both visuospatial and verbal memory traces are affected by the passage of time (Vergauwe et al., 2010), we predicted poorer recall performance in the word multiplication condition.

\section{Experiment 2}

Participants

Thirty-two undergraduate students (30 females; mean age = 21.1 years, $S D=2.8$ ) at the University of Geneva participated for partial course credit.

Materials and procedure

This second experiment used the same procedure as Experiment 1, except that participants memorized not five letters, but 3 out of 12 spatial locations defined by squares presented at fixed random locations on-screen. Each spatial location was presented by turning one of these squares blue for 1,000 ms, followed by three multiplications. In the recall phase, participants were presented with the 12 squares and had to click on the squares they had seen before in correct order. Each time participants clicked on a square, it turned blue. When participants had clicked on 3 squares, the experiment proceeded.

Results

Two participants who had fewer than $80 \%$ correct responses in the multiplication task were discarded from the analyses. As in the first experiment, it took longer to verify multiplications presented in words than those presented in digits $(1,279 \mathrm{~ms}$, $S D=192 \mathrm{~ms}$, and $908 \mathrm{~ms}, S D=100 \mathrm{~ms}$, respectively), $F(1,29)=268.24, p<.001$. These longer processing times resulted in a lower rate of series correctly recalled $(.58, S D=$ .27 , and $.73, S D=.19$, for the word and digit conditions, respectively), $F(1,29)=15.78, p<.01$. However, as in the first experiment, word multiplications involved more errors $(8 \%, S D=4 \%)$ than did digit multiplications $(5 \%, S D=$ $3 \%), F(1,29)=21.90, p<.001$. Because the rate of errors was lower than in the first experiment, it was possible to keep only the trials on which no error was committed on the arithmetic task. Those participants who had fewer than 3 remaining trials in one or both conditions were discarded from the analysis. The 25 remaining participants had a mean of 4.3 trials in the word condition and 5.5 trials in the digit condition, with mean response times of $1,274 \mathrm{~ms}(S D=188 \mathrm{~ms})$ and $893 \mathrm{~ms}(S D=104 \mathrm{~ms})$ for the word and digit conditions, respectively, $F(1,24)=274.04, p<.001$. Although the remaining trials did not involve any error on the arithmetic task, the word condition still involved more forgetting of spatial information than did the digit condition (rates of series correctly recalled of .63, $S D=.29$, and $.84, S D=.20$, respectively), $F(1,24)=18.63, p<.001$, an effect that remained significant even when the order of recall was not taken into account $(.68, S D=.27$, and $.87, S D=.19$, respectively), $F(1,24)=15.40, p<.001$.

\section{General discussion}

In two experiments, we observed that increasing the time during which attention was occupied by a concurrent processing activity while the time available for refreshing memory traces was kept constant resulted in lower recall performance. Most important, this result was observed for both verbal and visuospatial memory, whereas the intervening tasks remained unchanged, a result difficult to reconcile with interference accounts of forgetting. Indeed, if it can be assumed that

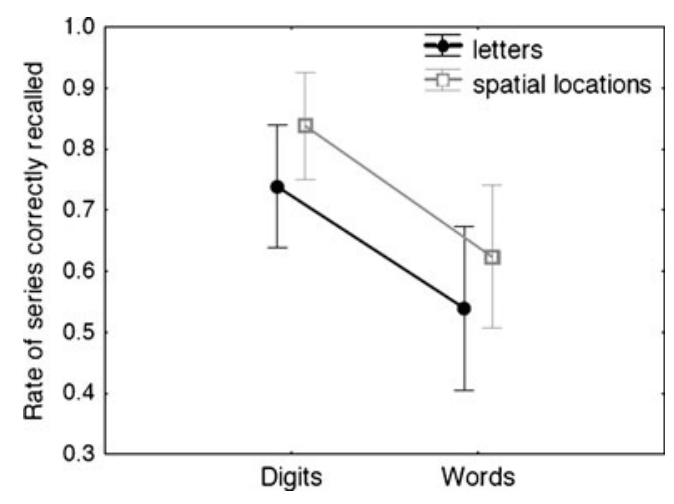

Fig. 2 Rate of series of letters and spatial locations correctly recalled as a function of the format of presentation of the multiplications 
encoding number words activates verbal representations susceptible to interference with letters to a greater extent than does encoding digits, it is more difficult to imagine that solving multiplications presented in word format involves more visuospatial representations than does solving the same multiplications in digit format. Seitz and Schumann-Hengsteler (2000), who studied the role of working memory resources in mental multiplication, observed that a visuospatial tapping task has no effect at all in solving multiplications, suggesting that solving small multiplications, such as those we used here, does not involve visuospatial representations. Thus, an interference account of forgetting would not predict that solving multiplications presented in word format should have a greater effect on the maintenance and recall of visuospatial information than solving these operations presented in digit format. On the contrary, we observed that processing word multiplications still induced more forgetting and lower recall performance even when visuospatial information was to be memorized. When error rates were equalized, the size of the effect induced by the longer processing times in the word condition was equivalent in both experiments (from .54 to .74 with verbal memoranda and from .63 to .84 with visuospatial memoranda; Fig. 2). The total absence of an interaction between the format of the intervening task (either words or digits) and the nature of the memoranda (either letters or spatial locations) supports the hypothesis that memory traces, whatever their nature, suffer from a temporal decay as long as attention is diverted by concurrent activities, as is assumed by the TBRS model of working memory (Barrouillet et al., 2004, 2007; Barrouillet, Portrat, \& Camos 2011; Vergauwe et al., 2009; Vergauwe et al., 2010). Our findings also mesh with and extend recent empirical findings suggesting that at least some features of memory traces decay with time (Barrouillet, Portrat, Vergauwe, Diependaele, \& Camos, 2011; Cowan \& AuBuchon, 2008; Ricker \& Cowan, 2010).

A possible alternative account would be to assume that the longer periods of attentional capture elicited by the word multiplications and the longer retention interval they involve would create more proactive interference. Such a hypothesis predicts that recall performance would progressively decline with tests, which determine the number of prior interfering associations, with a stronger decline in the word condition (Keppel \& Underwood, 1962). To test this hypothesis, we compared in both experiments recall performance on the first four and the last four trials in each condition. Contrary to the proactive interference hypothesis, the difference in recall between the two conditions did not significantly vary from the first to the last part of the experiments and was even slightly smaller on the last 4 trials, in Experiment 1 (rates of .43 and .71 of series correctly recalled in the word and digit conditions, respectively, over the first four trials, as compared with .58 and .75 over the last four trials), $F(1,18)=1.03$, $p=.32$, as well as in Experiment 2 (.53 and .71 in the word and digit conditions, respectively, over the first four trials, as compared with .65 and .80 for the last four trials), $F<1$. In fact, differences between conditions were present from the very first trial (.32 and .68 for the word and digit conditions, respectively, in Experiment 1, and .40 and .60, respectively, in Experiment 2). The facts that recall performance did not decline with tests, that the difference between conditions remained unchanged over trials, and that it was present on the very first trial do not fit very well with a proactive interference account.

Another alternative explanation would be to assume that forgetting depends not on temporal decay, but on the amount of attention used by the concurrent activity within a working memory in which processing and storage activities would be performed in parallel and fueled by a limited amount of shared attention. Word multiplications might use attention more intensively, resulting in poorer recall. However, we have shown elsewhere that intervening processing in complex span tasks have a detrimental effect on storage commensurate with their duration, whatever their nature (Barrouillet et al., 2007; Barrouillet, Portrat, \& Camos, 2011).

Overall, the simplest way to explain the present findings is to assume that memory traces decay with time when attention is not available to counteract temporal decay. This is not to say that representation-based interference does not play any role in forgetting, and we have already suggested that both temporal decay and representation-based interference could concur to produce forgetting from working memory (Barrouillet et al., 2007). Nonetheless, the present results add to the growing body of evidence that working memory strongly depends on time-related factors and constitute a further evidence for temporal decay in the short term, suggesting that the causal effect of time on forgetting cannot be easily dismissed.

Acknowledgements This research was supported by Grants 100014 122626 and 100014-132037 from the Swiss National Science Foundation to Pierre Barrouillet. The authors would like to thank Pr. Valérie Camos for comments on earlier drafts of the manuscript.

\section{References}

Barrouillet, P., Bernardin, S., \& Camos, V. (2004). Time constraints and resource-sharing in adults' working memory spans. Journal of Experimental Psychology: General, 133, 83-100.

Barrouillet, P., Bernardin, S., Portrat, S., Vergauwe, E., \& Camos, V. (2007). Time and cognitive load in working memory. Journal of Experimental Psychology: Learning, Memory, and Cognition, 33, $570-585$.

Barrouillet, P., Portrat, S., \& Camos, V. (2011). On the law relating processing to storage in working memory. Psychological Review, 118, 175-192.

Barrouillet, P., Portrat, S., Vergauwe, E., Diependaele, K., \& Camos, V. (2011). Further evidence for temporal decay in working memory: Reply to Lewandowsky and Oberauer (2009). Journal of 
Experimental Psychology: Learning, Memory, and Cognition, 37, $1302-1317$.

Brown, J. (1958). Some tests of the decay theory of immediate memory. Quarterly Journal of Experimental Psychology, 10, 12-21.

Brown, G. D. A., \& Lewandowsky, S. (2010). Forgetting in memory models: Arguments against trace decay and consolidation failure. In S. Della Salla (Ed.), Forgetting (pp. 49-76). Hove: Psychology Press.

Conrad, R. (1967). Interference or decay over short retention intervals. Journal of Verbal Learning and Verbal Behavior, 6, 49-54.

Cowan, N., \& AuBuchon, A. M. (2008). Short-term memory loss over time without retroactive stimulus interference. Psychonomic Bulletin \& Review, 15, 230-235.

Darling, S., Della Sala, S., \& Logie, R. H. (2007). Behavioural evidence for separating components within visuo-spatial working memory. Cognitive Processes, 8, 175-181.

Dehaene, S. (1992). Varieties of numerical abilities. Cognition, 44, 1-42.

Fias, W., Brysbaert, M., Geypens, F., \& d'Ydewalle, G. (1996). The importance of magnitude information in numerical processing: Evidence from the SNARC effect. Mathematical Cognition, 2, 95-110.

Jentzsch, I., \& Dudschig, C. (2008). Why do we slow down after an error? Mechanisms underlying the effect of post-error slowing. Quarterly Journal of Experimental Psychology, 62, 209-218.

Keppel, G., \& Underwood, B. J. (1962). Proactive inhibition in shortterm retention of single items. Journal of Verbal Learning and Verbal Behavior, 1, 153-161.

Laming, D. (1979). Choice reaction time performance following an error. Acta Psychologica, 43, 199-224.

Lewandowsky, S., \& Oberauer, K. (2009). No evidence for temporal decay in working memory. Journal of Experimental Psychology: Learning, Memory, and Cognition, 35, 1545-1551.
Lewandowsky, S., Oberauer, K., \& Brown, G. D. A. (2009). No temporal decay in verbal short-term memory. Trends in Cognitive Sciences, 13, 120-126.

Logie, R. H. (1995). Visuo-spatial working memory. Hove: Erlbaum.

Nairne, J. S. (1990). A feature model of immediate memory. Memory \& Cognition, 18, 251-269.

Noel, M. P., Fias, W., \& Brysbaert, M. (1997). About the influence of the representation format on arithmetical-fact retrieval processes. Cognition, 63, 335-374.

Oberauer, K., \& Kliegl, R. (2006). A formal model of capacity limits in working memory. Journal of Memory and Language, 55, 601-626.

Oberauer, K., \& Lewandowsky, S. (2008). Forgetting in immediate serial recall: Decay, temporal distinctiveness, or interference? Psychological Review, 115, 544-576.

Peterson, L. R., \& Peterson, M. J. (1959). Short-term retention of individual verbal items. Journal of Experimental Psychology, 58, 193-198.

Ricker, T. J., \& Cowan, N. (2010). Loss of visual working memory within seconds: The combined use of refreshable and nonrefreshable features. Journal of Experimental Psychology: Learning, Memory, and Cognition, 36, 1355-1368.

Seitz, K., \& Schumann-Hengsteler, R. (2000). Mental multiplication and working memory. European Journal of Cognitive Psychology, 12, 552-570.

Vergauwe, E., Barrouillet, P., \& Camos, V. (2009). Visual and spatial working memory are not that dissociated after all: A time-based resource-sharing account. Journal of Experimental Psychology: Learning, Memory, and Cognition, 35, 1012-1028.

Vergauwe, E., Barrouillet, P., \& Camos, V. (2010). Do mental processes share a domain-general resource? Psychological Science, 21, 384-390. 